\title{
Molecular Mechanism Linking BRCA1 Dysfunction to High Grade Serous Epithelial Ovarian Cancers with Peritoneal Permeability and Ascites
}

Desai $\mathrm{A}^{4}$, Xu J ${ }^{1}$, Aysola $\mathrm{K}^{1}$, Akinbobuyi $\mathrm{O}^{2}$, White $\mathrm{M}^{1}$, Reddy $\mathrm{VE}^{5}$, Okoli ${ }^{3}$, Clark $\mathrm{C}^{3}$, Partridge $\mathrm{EE}^{6}$, Childs $\mathrm{Ed}^{3}$, Beech $\mathrm{DJ}^{3}$, Rice $\mathrm{MV}^{1}$, Reddy ESP ${ }^{1}$ and Rao $\mathrm{VN}^{* 1}$

${ }^{1}$ Cancer Biology Program, Department of OB/GYN

${ }^{2}$ Department of Internal Medicine

${ }^{3}$ Department of Surgery, Morehouse School of Medicine, Georgia Cancer Center for Excellence, Grady Health System, Atlanta, USA

${ }^{4}$ Department of Internal Medicine, University of Buffalo, Erie County Medical Center, Buffalo NY

${ }^{5}$ Philadelphia College of Osteopathic Medicine, Suwanee GA

${ }^{6}$ Division of Gynecological Oncology, Department of Obstetrics and Gynecology, University of Alabama at Birmingham, Birmingham, Alabama

${ }^{*}$ Corresponding author: Rao VN, Professor, Co-Director of the Cancer Biology Program, GCC Distinguished Cancer Scholar, Department of OB/GYN, Morehouse School of Medicine. Georgia Cancer Center for Excellence, Rm 10C011, Grady Health System, 80 Jesse Hill Jr. Drive, Atlanta, Georgia 30303-3031, Fax: 404489-9220, Tel: 404-489-9993, E-mail: vrao@msm.edu

Citation: Desai A, Xu J, Aysola K, Akinbobuyi O, White M, et al. (2015) Molecular Mechanism Linking Brca1 Dysfunction to High Grade Serous Epithelial Ovarian Cancers with Peritoneal Permeability and Ascites. J Gynecol Res 1(1): 103. doi: 10.15744/2454-3284.1.103

Received Date: February 14, 2015 Accepted Date: April 22, 2015 Published Date: April 24, 2015

\begin{abstract}
Ovarian cancer constitutes the second most common gynecological cancer with a five-year survival rate of $40 \%$. Among the various histotypes associated with hereditary ovarian cancer, high-grade serous epithelial ovarian carcinoma (HGSEOC) is the most predominant and women with inherited mutations in BRCA1 have a lifetime risk of 40-60\%. HGSEOC is a challenge for clinical oncologists, due to late presentation of patient, diagnosis and high rate of relapse. Ovarian tumors have a wide range of clinical presentations including development of ascites as a result of deregulated endothelial function thereby causing increased vascular permeability of peritoneal vessels. The molecular mechanisms remain elusive. Studies have shown that fallopian tube cancers develop in women with BRCA1 gene mutations more often than previously suspected. Recent studies suggest that many primary peritoneal cancers and some high-grade serous epithelial ovarian carcinomas actually start in the fallopian tubes. In this article we have addressed the molecular pathway of a recently identified potential biomarker Ubc9 whose deregulated expression due to BRCA1 dysfunction can result in HGSEOC with peritoneal permeability and formation of ascites. We also discuss the role of downstream targets Caveolin-1 and Vascular Endothelial Growth Factor (VEGF) in the pathogenesis of ascites in ovarian carcinomas. Finally we hypothesize a signaling axis between Ubc9 over expression, loss of Caveolin-1 and induction of VEGF in BRCA1 mutant HGSEOC cells. We suggest that Ubc9-mediated stimulation of VEGF as a novel mechanism underlying ovarian cancer aggressiveness and ascites formation. Agents that target Ubc9 and VEGF signaling may represent a novel therapeutic strategy to impede peritoneal growth and spread of HGSEOC.
\end{abstract}

Keywords: BRCA1; BRCA1a; Ubc9; High Grade Serous Epithelial Ovarian Cancer; Caveolin-1; VEGF; Tumor suppression; Endothelial function; Ascites; Peritoneum; Fallopian tubes

\section{Introduction}

Ovarian cancer is a common gynecological cancers and the fifth leading cause of death in women. This year alone, about 22,000 women will be diagnosed with ovarian cancer and more than half will succumb to it [1]. Although there has been a lot of research in Ovarian cancer, efforts to decrease the mortality rate have not been successful due to lack of biomarkers for early detection and also the origin and pathogenesis of ovarian cancer are not well-understood [2]. Hereditary ovarian cancers are associated with various mutations, the most common of which are in the tumor suppressor genes BRCA1 and BRCA2. For carriers of BRCA1 mutations, the risk of ovarian cancer is about 50\% [3]. Recent studies suggest that ovarian cancer should no longer be considered as a single entity. Many studies in which the fallopian tubes were carefully examined revealed that in situ and small early invasive tubal carcinomas occurred in women with a genetic predisposition for the development of ovarian cancer [2,4-7]. Thus, fallopian tube carcinoma is now included in the spectrum of cancers associated with BRCA mutations [8]. This study lends further evidence to the above hypothesis. They proposed that cancer cells are shed from the tubal epithelium and consequently get implanted on the surface of the ovary to form inclusion cysts to produce ovarian or primary peritoneal cancers [8]. Ovarian cancer cells usually 
disseminate by intraperitoneal seeding where tumor cells are transported by the peritoneal fluid and implant into the mesothelial lineages of the cavity wall. This is mediated by cross-talk between tumor and stromal cells are often accompanied with ascites [9]. Hereditary ovarian cancers are associated with many mutations. The most common among them are mutations in the tumor suppressor genes BRCA1 and BRCA2. The risk of ovarian cancer with BRCA1 mutation carriers is as high as 50\% [3].

\section{Caveolin}

Recently there have been a number of efforts towards identifying new biomarkers expressed in ovarian cancers. Caveolin-1 is one such protein which has been associated with ovarian cancer. Caveolae are non-Clathrin flask shaped 50-100 nm cell surface invaginations that participate in cellular functions like signal transduction, endocytosis and vesicular transport. Caveolins are important proteins responsible for the structure of caveolae. Overexpression of Caveolin-1 in cells, which do not usually express Caveolae (lymphocytes), results in formation of Caveolae and down regulation of Caveolin-1 in Caveolin rich cells leading to the loss of these membrane invaginations. The above lends evidence to the close correlation between Caveolin and Caveolae [10-13].

There are 3 different Caveolin proteins: Caveolin 1 (Cav-1), Caveolin 2 (Cav-2) and Caveolin 3 (Cav-3). Cav-1 and Cav-2 are found abundantly in the endothelial cells, fibroblasts, smooth muscle cells and adipocytes, while Cav-3 is mainly expressed in skeletal and cardiac musculature. Cav-1 has two isoforms: (a) the alpha isoform consisting of 178 amino acids and (b) the beta isoform truncated by 32 amino acids at the $\mathrm{N}$-terminus. Caveolin has a hair-pin structure with the $\mathrm{C}$ and $\mathrm{N}$ terminals positioned towards the cytosol. It consists of an oligomerization domain and a scaffolding domain with the latter being responsible for interaction with signaling proteins [14]. Membrane attachment is made possible by the $\mathrm{N}$-and-C terminal domains, the transmembrane domain and the palmitoyl groups at the C-terminus $[15,16]$. Caveolins have a tendency to form oligomers which interact with other caveolin-1 oligomers at the C-terminal domains forming a meshwork responsible for the formation of invaginations in the plasma membrane [17].

As mentioned above, caveolae are important components of the cell membrane which are important for many cellular processes. We list some of them below --

Transcytosis: A mechanism of caveolae mediated vesicular trafficking. Schnitzer et al. [18] used antibodies targeting the extracellular domains of proteins in endothelial caveolin proteins and showed that the antibodies were transported directly through the caveolae across the capillary wall.

Endocytosis: Montesano et al. [19] first demonstrated that caveolae bind and internalize cholera and tetanus toxins. This theory was strengthened by Schnitzer et al. [20] who demonstrated that treatment of cells with cholesterol binding agents resulted in abolition of caveolin mediated endocytosis of albumin sparing the clathrin dependent process. Purification of caveolae has shown that the components used by cells in vesicle formation are concentrated in caveolae and likely associated with Cav-1 [21-23]

Cholesterol and Caveolin: Caveolae expression is dependent on the tissue concentration of cholesterol [24]. Treatment with cholesterol binding agents is associated with flattening of these pits [25]. In-vitro studies showed a direct correlation between cholesterol and caveolin when purified caveolin-1 was found to reconstitute only into lipid vesicles containing cholesterol [26]. Caveolae play an important role in cholesterol removal from the cells [27] and intracellular transport of newly synthesized cholesterol [28].

Signalosomes: The Caveolae/Raft signaling hypothesis was proposed by Lisanti et al. [29] when they found signal transduction molecules on biochemically separating the caveolar membrane. The scaffolding domain of caveolins interact with and influence the activity of signaling molecules present on the caveolae thus proving that caveolae function has as specialized signaling organelles or signalosomes.

Oncogenesis: Various studies demonstrated down-regulation of Cav-1 by many tumor cells suggesting that Cav-1 is a target of the activated oncogenes. Stable expression of H-Ras in the NIH 3T3 cell line led to down-regulation of Cav-1 [30] whereas Engelman et al. [11] showed that treatment of these cells with MAP kinase inhibitors led to increased Cav-1 levels. The q31 region on human chromosome 7 has particular importance in the development of many primary tumors including but not limited to breast [31] and ovarian tumors [32] due to the presence of high frequency deletions around the D7S522 CA-repeat microsatellite region, which is a marker mapping the 7p31.1 region [31,33]. Lisanti et al. [29] showed the close proximity of Cav-1 and Cav-2 to q31.1 region of human chromosome 7 thus lending more evidence to the hypothesis that Cav-1 functions as a tumor suppressor gene [34]. Loss of Cav-1 expression was shown in serous ovarian carcinomas [35]. In fact expression of Cav-1 in ovarian cancer cells resulted in suppression of tumor cell survival in vitro, suggesting that Cav-1 may act as a tumor suppressor [35].

Angiogenesis: It is the creation of new blood vessels from preexisting vessels which is necessary for the formation of granulation tissue, wound healing and also an important component of tumor growth. VEGF is integrally linked with vascular permeability and angiogenesis [36,37] it was shown that tumor microvasculature is fenestrated and that topical administration of VEGF-165 resulted in the formation of fenestrations in the endothelium of vessels and also resulted in the formation of clusters of Caveoli as part of the angiogenic response. Liu et al. [38] further showed a positive correlation between Cav-1 expression and the formation of capillary tubes. Whereas, down regulation of Cav-1 by an anti-sense adenoviral approach resulted in a 10 fold decrease in the formation of capillary tubules proving the central role of Caveoli in angiogenesis. 


\section{Caveolae}

Cav-1 is found to be maximally concentrated in the endothelium [29]. Caveolin's role in regulating vascular permeability is multifold. Firstly, Cav-1 plays an important role in regulating Nitric Oxide levels in the endothelium. The scaffolding domain of Cav-1 binds to and is responsible for inhibiting endothelial Nitric Oxide synthase [39]. Bucci et al. [40] studied this using a mouse inflammation model and showed that the addition of the scaffolding domain of Cav-1 reduced the vascular permeability and tissue edema effect of Nitric Oxide. Based on the above, one may hypothesize that Cav-1, by regulating vascular permeability, reduces tumor progression in a mouse model [41].

Secondly, Cav-1 plays an important role in protein interactions between the cell and the extracellular matrix thus affecting permeability. Electron microscopic analysis of Cav-1 null mice revealed defects in tight endothelial junction formation and poor capillary endothelial cell adhesion to the basement membrane [42]. Endothelial permeability depends on the size of the molecule and the different receptors present within the caveolae. For example VEGF can cause grouping of caveolae into organelles [43]. Exposure of endothelial cells to VEGF caused increased formation of fenestrations [44].

Ovarian carcinoma has a wide range of clinical presentations including but not limited to ascites and peritoneal metastasis. The pathophysiology of ascites in malignancy is complex. In case of advanced cancers, the ascites can be caused by increased tumor burden blocking the drainage of lymphatic fluid. Some of the other mechanisms of ascites in malignancy include increased permeability of the vasculature.

\section{Vascular Endothelial Growth Factor}

VEGF has been implicated in tumor growth and metastasis due to its role in neovascularization and increasing vascular permeability. Mesiano et al. investigated the role of VEGF in ovarian carcinoma using in vivo models. They induced intraperitoneal tumors in immunodeficient mice with the human ovarian cancer cell line SKOV-3. Some of the mice were then treated with A4.6.1 which is a VEGF antibody and were compared to controls. Results showed that mice treated with anti-VEGF antibody had significantly decreased tumor growth compared with controls. On the other hand, once treatment with A4.6.1 was discontinued, these mice rapidly developed ascites. This stresses the importance of VEGF in ascites formation and intraperitoneal tumor growth [45]

Santin et al. [46] quantified the amount of VEGF secreted in many fresh and established ovarian cancer cell lines using ELISA. They found that the amount of VEGF secreted was directly proportional to the grade of the tumors with high grade tumors secreting high levels of VEGF compared to lower grade tumors. Further, fresh tumor cell lines were found to secrete more VEGF compared to established cancer cell lines. The ascitic fluid in ovarian cancer patients also had increased levels of VEGF compared to samples from healthy subjects. Therefore, VEGF plays a crucial role in regulating the endothelial cell function in the vasculature influencing many processes like angiogenesis, tumorigenesis and mediating malignancy associated ascites. It has also been found that serum VEGF levels correlate with clinical stage, the degree of metastasis and survival with increased VEGF levels are associated with poor survival (35 months versus 28 months) [47].

However it is not clear if VEGF alone can cause ascites and if drugs targeting VEGF can prevent metastatic disease. To further substantiate the above, Byrne et al., used retroviruses to generate a population of human SKOV-3 carcinoma cells to overproduce murine-VEGF and the peritoneal cells were directly transduced with adenovirus to produce VEGF. They showed that the formation of ascites is markedly increased by the overexpression of VEGF in both the mice injected SKOV3 carcinoma cells and in the peritoneum of mice lacking tumor invasion. They used a VEGF-trap, which is a high affinity soluble decoy receptor consisting of extracellular domains of both VEGFR1 and VEGFR2 and reported inactivation of VEGF with inhibition of ascites formation and decreased tumor burden in the mouse models [48]. Recently, Lei Yu et al. reported poor progression free survival and overall survival in ovarian cancer patients with increased tissue and serum VEGF expression in a meta-analysis of 16 studies analyzing 111 patients $[49,50]$.

\section{UBC9 and BRCA1 Mutation}

Hereditary ovarian cancers are associated with mutations in the tumor suppressor genes BRCA1 and BRCA2 [51,52]. BRCA1 is a nuclear-cytoplasm shuttling protein involved in multiple biological processes including DNA damage repair, cell growth, apoptosis, transcription activation or repression etc. [52]. We have previously shown that the tumor suppressor gene BRCA1 and its isoform BRCA1a to inhibit the tumor growth of ovarian cancer cells [53] by tethering Ubc9, a SUMO conjugating enzyme [54]. Ubc9 was found to be expressed at high levels in BRCA1 mutant HGSEOC cells [54] and it promotes cell invasion and metastasis of breast cancer cells implicating a role in tumorigenesis [55]. BRCA1 also regulates the expression of Caveolin-1 and inhibits the invasiveness and metastatic potential of mouse embryo fibroblasts [56]. BRCA1 also inhibits VEGF transcriptional activation and protein secretion through the estrogen signaling pathway in breast cancer cells [57]. We have previously shown BRCA1 proteins unlike its disease associated mutant BRCA1 proteins to bind Ubc9 and induce the expression of Caveolin-1 in BRCA1 mutant HGSEOC cells UWB1.289 [58]. Caveolin-1 is known to regulate VEGF levels thus maintaining endothelial permeability and normal ovarian function. We have observed elevated levels of VEGF in UWB1.289 compared to BRCA1 transfected UWB1.289 cells on using VEGF antibody by immunofluorescence analysis (Figure 1). In a recent study high level of Ang-1, Ang-2 and VEGF expression was observed in both BRCA carriers and TNBC patients suggesting VEGF as an attractive angiogenic target for these 
cancers [59]. As previously discussed, Caveolin-1 plays a major role in vascular permeability. Decreased levels of Caveolin-1 cause increased vascular permeability, thus increasing expression of VEGF which in turn result in the generation of tumor ascites as seen in advanced stages of ovarian cancers (Figure 2).

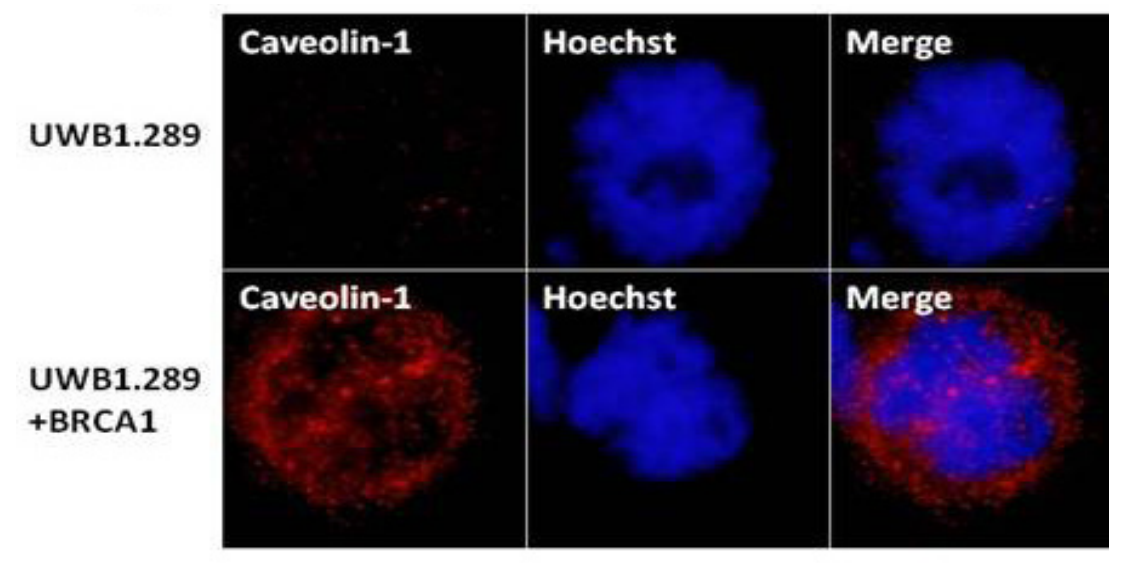

B

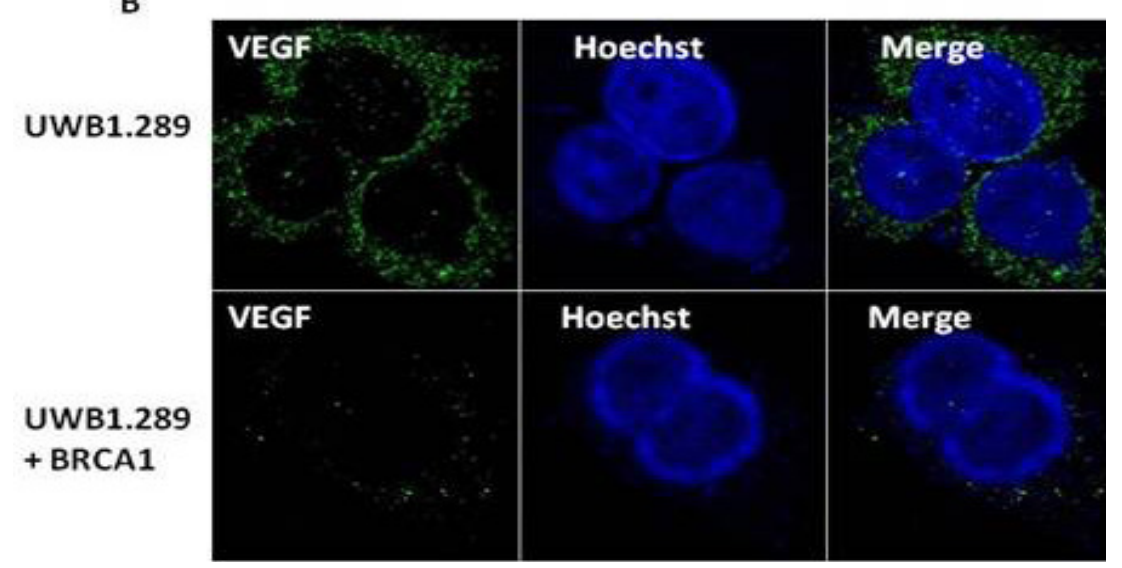

Figure 1: Loss of Caveolin-1 and induction of VEGF expression in BRCA1 mutant HGSEOC cells UWB1.289 as detected by immunofluorescence analysis. UWB1.289 and UWB1.289 + BRCA1 cells were seeded into six-well plates. The nuclei were visualized with DNA stain Hoechst 33258. Cells were fixed in methanol and probed with A: Caveolin-1(Santa Cruz, 1/250) B: VEGF(Santa Cruz, 1/250) followed by Alexa Fluor 568/488 labeled secondary antibody(Invitrogen, 1/200) staining as described (54). The images were taken using fluorescent microscope (100X, oil Olympus)
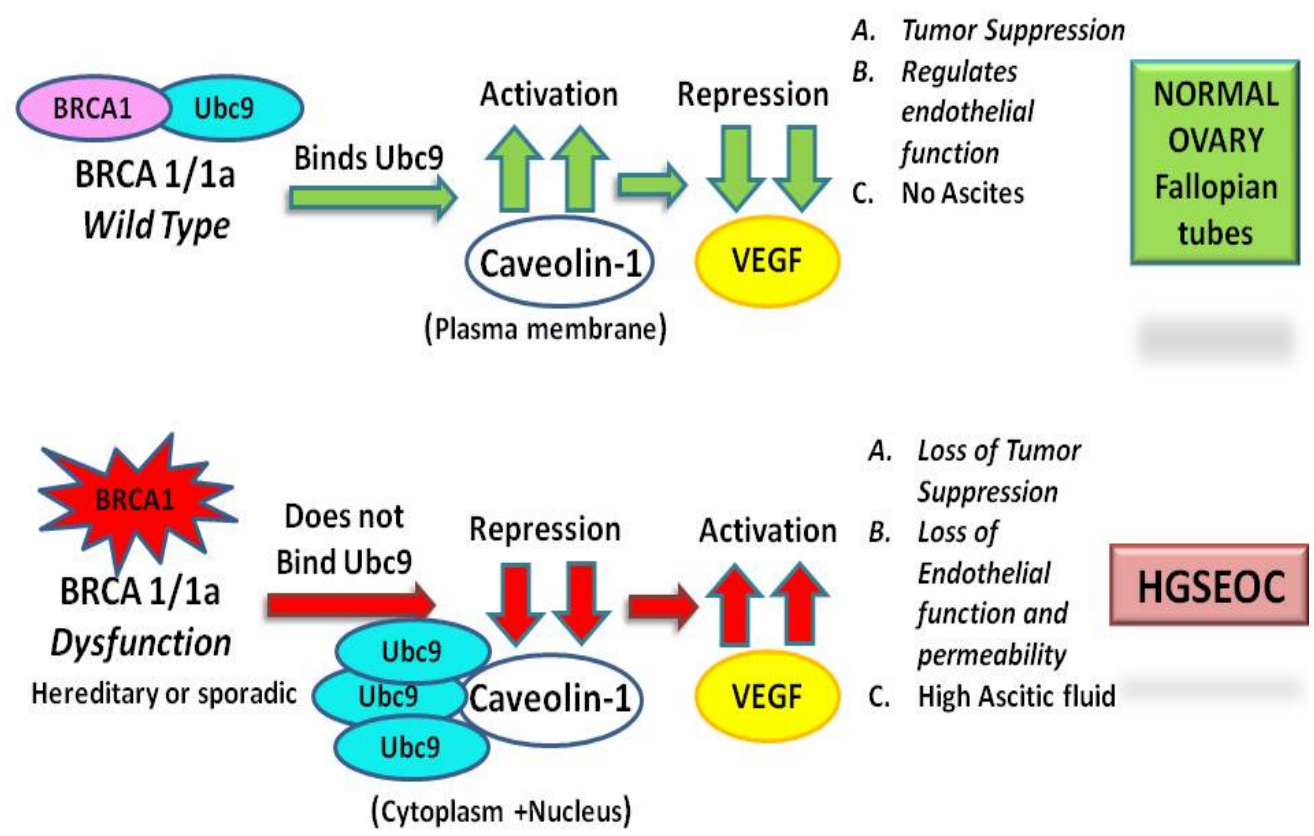

Figure 2: Working hypothetical model showing how BRCA1/1a binding to Ubc9 induces Caveolin-1 expression, down regulates VEGF regulates endothelial function in normal ovaries and fallopian tubes. In HGSEOC with BRCA1 dysfunction, Ubc9 is unleashed which inhibits Caveolin-1 expression causing increased levels of VEGF, loss of endothelial function and accumulation of ascites due to dissemination of cancer cells to the peritoneal cavity resulting in HGSEOC 


\section{Conclusion}

In conclusion our hypothesis suggests a molecular mechanism of BRCA1 dysfunction in HGSEOC with peritoneal metastasis and ascites. This review offers opportunities to better understand the origin and mechanism of development of BRCA1 mutant HGSEOC (Figure 3) which should lead to the development of more effective biomarkers for early detection as well as targeted therapies for BRCA1 associated HGSEOC.

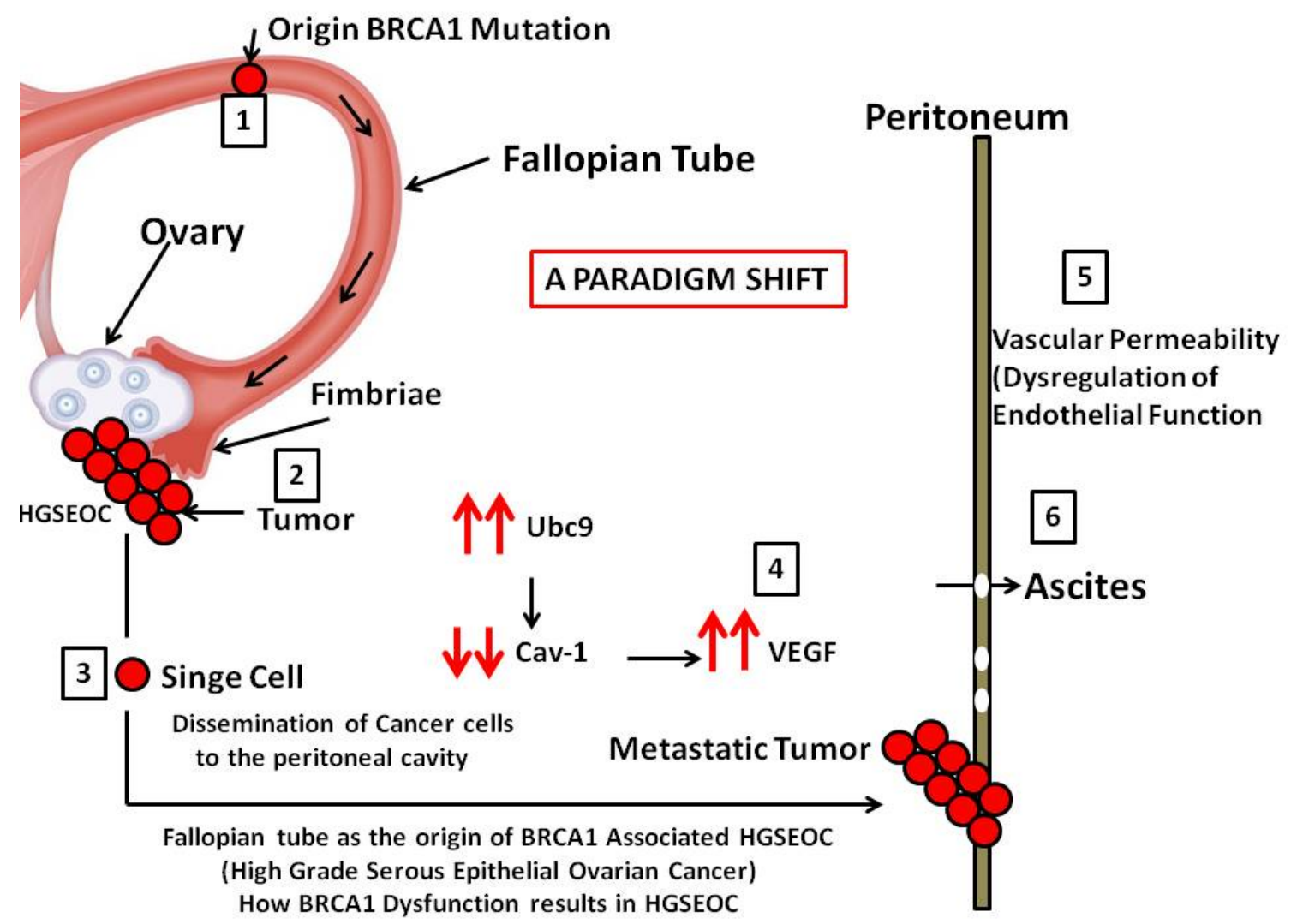

Figure 3: This is a simple model showing how HGSEOC initiates in the fallopian tube of women carrying a BRCA1 mutation. According to this scenario, the cancer can start in the fallopian tubes and the cells get detached and adhere to the ovarian surface epithelial where tumor develops resulting in deregulated levels of Ubc9, low levels of Caveolin-1 and excess VEGF. This triggers dissemination of cancer cells to the peritoneal cavity, tumor metastasis, increased vascular permeability and formation of ascites

\section{Acknowledgement}

We thank Mr. Abramson, research media services, Ms. Wimes and Dr. Donald Hill for editorial assistance. This work was supported in part by Georgia Cancer Coalition Distinguished Cancer Scholar award, NIH-NCRR-RCMI grant G-12-RR003034, U54 RR02613, 5P20RR11104, NIHMD research endowment grant 2S21MD000101 and U54 CA118638 to V.N.R. Georgia Cancer Coalition Distinguished Cancer Scholar award to E.S.P.R. V.N.R's lab was also supported in part by funds from the ING foundation.

\section{References}

1. Siegel RL, Miller KD, Jemal A (2015) Cancer Statistics 2015. CA Cancer J Clin 65: 5-29.

2. Bell DA, Scully RE (1994) Early de novo ovarian carcinoma. A study of fourteen cases. Cancer 73: 1859-64.

3. Shih IeM, Chen L, Wang CC, Gu J, Davidson B, et al. (2010) Distinct DNA methylation profiles in ovarian serous neoplasms and their implications in ovarian carcinogenesis. Am J Obstet Gynecol 203: 584.e1-22.

4. Salazar H, Godwin AK, Daly MB, Laub PB, Hogan WM, et al. (1996) Microscopic benign and invasive malignant neoplasms and a cancer-prone phenotype in prophylactic oophorectomies. J Natl Cancer Inst 88: 1810-20.

5. Meinhold-Heerlein I, Bauerschlag D, Hilpert F, Dimitrov P, Sapinoso LM, et al. (2005) Molecular and prognostic distinction between serous ovarian carcinomas of varying grade and malignant potential. Oncogene 24: 1053-65.

6. Gilks CB, Vanderhyden BC, Zhu S, van de Rign M, Longacre TA (2005) Distinction between serous tumors of low malignant potential and serous carcinomas based on global mRNA expression profiling. Gynecol Oncol 96: 684-94.

7. Norquist BM, Garcia RL, Allison KH, Jokinen CH, Kernochan LE, et al. (2010) The molecular pathogenesis of hereditary ovarian carcinoma: alterations in the tubal epithelium of women with BRCA1 and BRCA2 mutations. Cancer 116: 5261-71.

8. Shaw PA, Rouzbahman M, Pizer ES, Pintilie M, Begley H (2009) Candidate serous cancer precursors in fallopian tube epithelium of BRCA1/2 mutation carriers. Mod Pathol 22: 1133-8.

9. Nahora H (2014) Heterotypic cellular interactions in the ovarian tumor microenvironment: biological significance and therapeutic implications. Front Oncol 4: 18. 
10. Fra AM, Williamson E, Simons K, Parton RG (1995) De novo formation of caveolae in lymphocytes by expression of VIP21-caveolin. Proc Natl Acad Sci U S A 92: 8655-9.

11. Engelman JA, Wykoff CC, Yasuhara S, Song KS, Okamoto T, et al. (1997) Recombinant expression of caveolin-1 in oncogenically transformed cells abrogates anchorage-independent growth. J Biol Chem 272: 16374-81.

12. Li S, Galbiati F, Volonte D, Sargiacomo M Engelman JA, et al. (1998) Mutational analysis of caveolin-induced vesicle formation. Expression of caveolin-1 recruits caveolin-2 to caveolae membranes. FEBS Lett 434: 127-34.

13. Galbiati F, Volonte D, Engelman JA, Watanabe G, Burk R, et al. (1998) Targeted downregulation of caveolin-1 is sufficient to drive cell transformation and hyperactivate the p42/44 MAP kinase cascade. EMBO J 17: 6633-48.

14. Liu P, Rudick M, Anderson RG (2002) Multiple functions of caveolin-1. J Biol Chem 277: 41295-8.

15. Dietzen DJ, Hastings WR, Lublin DM (1995) Caveolin is palmitoylated on multiple cysteine residues. Palmitoylation is not necessary for localization of caveolin to caveolae. J Biol Chem 270: 6838-42.

16. Schlegel A, Lisanti MP (2000) A molecular dissection of caveolin-1 membrane attachment and oligomerization. Two separate regions of the caveolin-1 C-terminal domain mediate membrane binding and oligomer/oligomer interactions in vivo. J Biol Chem 275: 21605-17.

17. Razani B, Woodman SE, Lisanti MP (2002) Caveolae: from cell biology to animal physiology. Pharmacol Rev 54: $431-67$.

18. Schnitzer JE (2001) Caveolae: from basic trafficking mechanisms to targeting transcytosis for tissue-specific drug and gene delivery in vivo. Adv Drug Deliv Rev 49: 265-80.

19. Montesano R, Roth J, Robert A, Orci L (1982) Non-coated membrane invaginations are involved in binding and internalization of cholera and tetanus toxins. Nature 296: 651-3.

20. Schnitzer JE, Oh P, Pinney E, Allard J (1994) Filipin-sensitive caveolae-mediated transport in endothelium: reduced transcytosis, scavenger endocytosis, and capillary permeability of select macromolecules. J Cell Biol 127: 1217-32.

21. Schnitzer JE, Oh P, Jacobson BS, Dvorak AM (1995) Caveolae from luminal plasmalemma of rat lung endothelium: microdomains enriched in caveolin, Ca(2+)ATPase, and inositol trisphosphate receptor. Proc Natl Acad Sci U S A 92: 1759-63.

22. Schnitzer JE, McIntosh DP, Dvorak AM, Liu J, Oh P (1995) Separation of caveolae from associated microdomains of GPI-anchored proteins. Science 269: 14359.

23. Predescu SA, Predescu DN, Palade GE (2001) Endothelial transcytotic machinery involves supramolecular protein-lipid complexes. Mol Biol Cell 12: 1019-33.

24. Hailstones D, Sleer LS, Parton RG, Stanley KK (1998) Regulation of caveolin and caveolae by cholesterol in MDCK cells. J Lipid Res 39: 369-79.

25. Rothberg KG, Heuser JE, Donzell WC, Ying YS, Glenney JR, et al. (1992) Caveolin, a protein component of caveolae membrane coats. Cell 68: 673-82.

26. Murata M, Peranen J, Schreiner R, Wieland F, Kurzchalia TV, et al. (1995) VIP21/caveolin is a cholesterol-binding protein. Proc Natl Acad Sci USA 92: 10339-43.

27. Fielding CJ, Fielding PE (1997) Intracellular cholesterol transport. J Lipid Res 38: 1503-21.

28. Smart EJ, Ying Ys, Donzell WC, Anderson RG (1996) A role for caveolin in transport of cholesterol from endoplasmic reticulum to plasma membrane. J Biol Chem 271: 29427-35.

29. Lisanti MP, Scherer PE, Vidugiriene J, Tang Z, Hermanowski-Vosatka A, et al. (1994) Characterization of caveolin-rich membrane domains isolated from an endothelial-rich source: implications for human disease. J Cell Biol 126: 111-26.

30. Koleske AJ, Baltimore D, Lisanti MP (1995) Reduction of caveolin and caveolae in oncogenically transformed cells. Proc Natl Acad Sci U S A 92: 1381-5.

31. Zenklusen JC, Bièche I, Lidereau R, Conti CJ (1994) (C-A)n microsatellite repeat D7S522 is the most commonly deleted region in human primary breast cancer. Proc Natl Acad Sci USA 91: 12155-8.

32. Kerr J, Leary JA, Hurst T, Shih YC, Antalis TM, et al. (1996) Allelic loss on chromosome 7q in ovarian adenocarcinomas: two critical regions and a rearrangement of the PLANH1 locus. Oncogene 13: 1815-8.

33. Zenklusen JC, Weitzel JN, Ball HG, Conti CJ (1995) Allelic loss at 7q31.1 in human primary ovarian carcinomas suggests the existence of a tumor suppressor gene. Oncogene 11:359-63.

34. Engelman JA, XL Zhang, MP Lisanti (1998) Genes encoding human caveolin-1 and -2 are co-localized to the D7S522 locus (7q31.1), a known fragile site (FRA7G) that is frequently deleted in human cancers. FEBS Lett 436: 403-10.

35. Wiechen K, Diatchenko L, Agoulnik A, Scharff KM, Schober H, et al. (2001) Caveolin-1 is down-regulated in human ovarian carcinoma and acts as a candidate tumor suppressor gene. Am J Pathol 159: 1635-43.

36. Roberts WG, Palade GE(1995) Increased microvascular permeability and endothelial fenestration induced by vascular endothelial growth factor. J Cell Sci 108: 2369-79.

37. Roberts WG, Palade GE (1997) Neovasculature induced by vascular endothelial growth factor is fenestrated. Cancer Res 57: 765-72.

38. Liu J, Wang XB, Park DS, Lisanti MP (2002) Caveolin-1 expression enhances endothelial capillary tubule formation. J Biol Chem 277: 10661-8.

39. Minshall RD, Sessa WC, Stan RV, Anderson RG, Malik AB (2003) Caveolin regulation of endothelial function. Am J Physiol Lung Cell Mol Physiol 285: L117983.

40. Bucci M, Gratton JP, Rudic RD, Acevedo L, Roviezzo F, et al. (2000) In vivo delivery of the caveolin-1 scaffolding domain inhibits nitric oxide synthesis and reduces inflammation. Nat Med 6: 1362-7.

41. Lin MI, Yu J, Murata T, Sessa WC (2007) Caveolin-1-deficient mice have increased tumor microvascular permeability, angiogenesis, and growth. Cancer Res 67: 2849-56.

42. Schubert W, Frank PG, Woodman SE, Hyogo H, Cohen DE, et al. (2002) Microvascular hyperpermeability in caveolin-1 (-/-) knock-out mice. Treatment with a specific nitric-oxide synthase inhibitor, L-NAME, restores normal microvascular permeability in Cav-1 null mice. J Biol Chem 277: $40091-8$.

43. Vasile E, Qu-Hong, Dvorak HF, Dvorak AM (1999) Caveolae and vesiculo-vacuolar organelles in bovine capillary endothelial cells cultured with VPF/VEGF on floating Matrigel-collagen gels. J Histochem Cytochem 47: 159-67.

44. Chen J, Braet F, Brodsky S, Weinstein T, Romanov V, et al. (2002) VEGF-induced mobilization of caveolae and increase in permeability of endothelial cells. Am J Physiol Cell Physiol 282: C1053-63.

45. Mesiano S, Ferrara N, RB Jaffe (1998) Role of vascular endothelial growth factor in ovarian cancer: inhibition of ascites formation by immunoneutralization. Am J Pathol 153: 1249-56. 
46. Santin AD, Hermonat PL, Ravaggi A, Cannon MJ, Pecorelli S, et al. (1999) Secretion of vascular endothelial growth factor in ovarian cancer. Eur J Gynaecol Oncol 20: 177-81.

47. Li L, Wang L, Zhang W, Tang B, Zhang J, et al. (2004) Correlation of serum VEGF levels with clinical stage, therapy efficacy, tumor metastasis and patient survival in ovarian cancer. Anticancer Res 24: 1973-9.

48. Byrne AT, Ros L, Holash J, Nakanishi M, Hu L, et al. (2003) Vascular endothelial growth factor-trap decreases tumor burden, inhibits ascites, and causes dramatic vascular remodeling in an ovarian cancer model. Clin Cancer Res 9: 5721-8.

49. Yu L, Deng L, Li J, Zhang Y, Hu L (2013) The prognostic value of vascular endothelial growth factor in ovarian cancer: a systematic review and meta-analysis. Gynecol Oncol 128: 391-6.

50. Cheng D, B Liang, Y Li (2013) Serum vascular endothelial growth factor (VEGF-C) as a diagnostic and prognostic marker in patients with ovarian cancer. PLoS One 8: e55309.

51. Berchuck A, Heron KA, Carney ME, Lancaster JM, Fraser EG, et al. (1998) Frequency of germline and somatic BRCA1 mutations in ovarian cancer. Clin Cancer Res 4: 2433-7.

52. Desai A, Xu J, Aysola K, Qin Y, Okoli C, et al. (2014) Epithelial Ovarian Cancer: An Overview. World J Transl Med 3: 1-8.

53. Yuli C, Shao N, Rao R, Aysola P, Reddy V, et al. (2007) BRCAla has antitumor activity in TN breast, ovarian and prostate cancers. Oncogene 26: 6031-7.

54. Qin Y, Xu J, Aysola K, Oprea G, Reddy A, et al. (2012) BRCA1 Proteins regulate growth of ovarian cancer cells by tethering Ubc9. Am J Cancer Res 2: 540-8.

55. Zhu S, Sachdeva M, Wu F, Lu Z, Mo YY (2010) Ubc9 promotes breast cell invasion and metastasis in a sumoylation-independent manner. Oncogene 29: 1763-72. 56. Wang Y, Yu J, Zhan Q (2008) BRCA1 regulates caveolin-1 expression and inhibits cell invasiveness. Biochem Biophys Res Commun 370: 201-6.

57. Kawai H, Li H, Chun P, Avraham S Avraham HK (2002) Direct interaction between BRCA1 and the estrogen receptor regulates vascular endothelial growth factor (VEGF) transcription and secretion in breast cancer cells. Oncogene 21: 7730-9.

58. Xu J, Agyemang S, Qin Y, Aysola K, Giles M, et al. (2014) A Novel Pathway that Links Caveolin-1 Down-Regulation to BRCA1 Dysfunction in Serous Epithelial Ovarian Cancer Cells. Enliven Chall Cancer Detect Ther 1.

59. Danza K, Pilato B, Lacalamita R, Addati T, Giotta F, et al. (2013) Angiogenetic axis angiopoietins/Tie2 and VEGF in familial breast cancer. Eur J Hum Genet 21: 824-30.

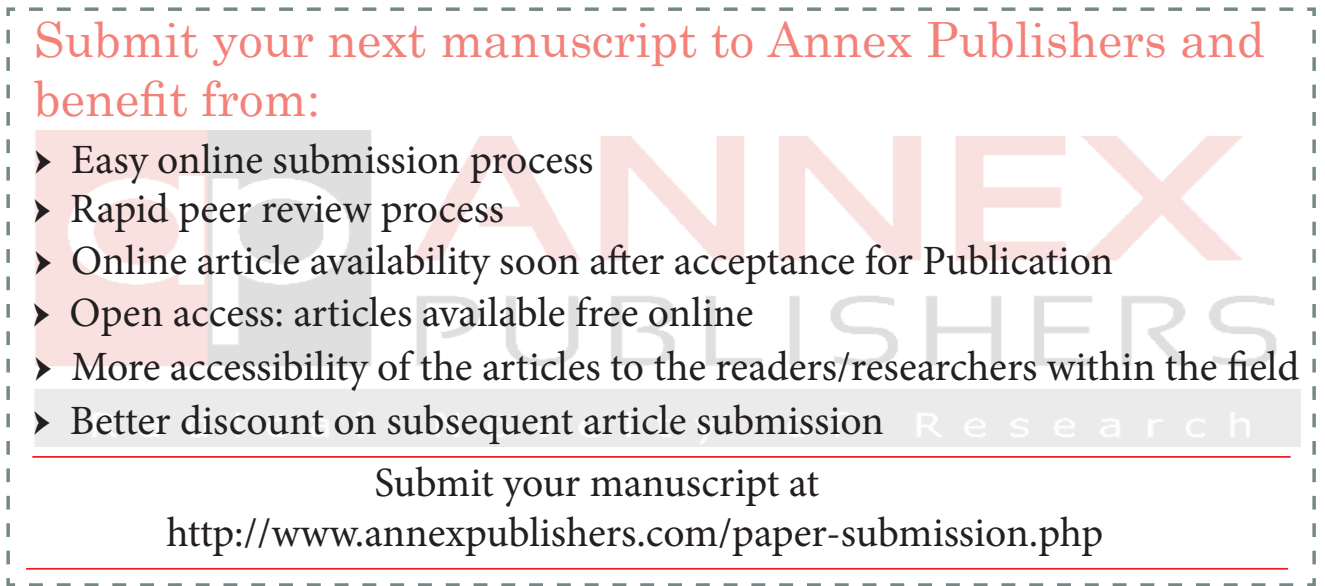

